\title{
Transmission properties of composite metamaterials in free space
}

Mehmet Bayindir, K. Aydin, E. Ozbay, P. Markoš, and C. M. Soukoulis

Citation: Appl. Phys. Lett. 81, 120 (2002); doi: 10.1063/1.1492009

View online: http://dx.doi.org/10.1063/1.1492009

View Table of Contents: http://aip.scitation.org/toc/apl/81/1

Published by the American Institute of Physics

\section{Articles you may be interested in}

Microwave transmission through a two-dimensional, isotropic, left-handed metamaterial

Applied Physics Letters 78, 489 (2001); 10.1063/1.1343489

Electric-field-coupled resonators for negative permittivity metamaterials

Applied Physics Letters 88, 041109 (2006); 10.1063/1.2166681

High performance optical absorber based on a plasmonic metamaterial

Applied Physics Letters 96, 251104 (2010); 10.1063/1.3442904

Origin of dissipative losses in negative index of refraction materials

Applied Physics Letters 82, 2356 (2003); 10.1063/1.1563726

Direct calculation of permeability and permittivity for a left-handed metamaterial

Applied Physics Letters 77, 2246 (2000); 10.1063/1.1314884

Capacitor-loaded split ring resonators as tunable metamaterial components

Journal of Applied Physics 101, 024911 (2007); 10.1063/1.2427110

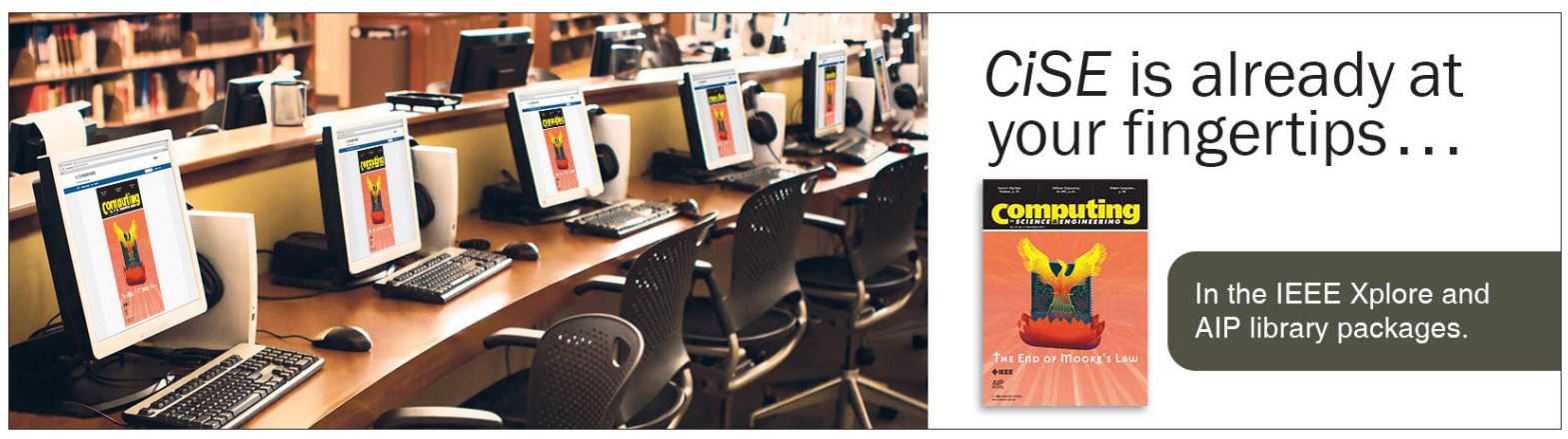




\title{
Transmission properties of composite metamaterials in free space
}

\author{
Mehmet Bayindir, ${ }^{\text {a) }}$ K. Aydin, and E. Ozbay \\ Department of Physics, Bilkent University, Bilkent, 06533 Ankara, Turkey \\ P. Markošb) and C. M. Soukoulis ${ }^{\mathrm{c})}$ \\ Ames Laboratory and Department of Physics, Iowa State University, Ames, Iowa 50011
}

(Received 31 January 2002; accepted for publication 13 May 2002)

\begin{abstract}
We propose and demonstrate a type of composite metamaterial which is constructed by combining thin copper wires and split ring resonators (SRRs) on the same board. The transmission measurements performed in free space exhibit a passband within the stop bands of SRRs and thin wire structures. The experimental results are in good agreement with the predictions of the transfer matrix method simulations. (C) 2002 American Institute of Physics. [DOI: 10.1063/1.1492009]
\end{abstract}

In recent years, the composite metamaterials (CMMs) have inspired great interest due to their unique physical properties and novel applications of these materials. ${ }^{1,2}$ Two important parameters, electrical permittivity $\epsilon$ and magnetic permeability $\mu$, determine the response of the material to the electromagnetic radiation. Generally, $\epsilon$ and $\mu$ are both positive in ordinary materials. While $\epsilon$ could be negative in ordinary materials (for instance in metals), no natural materials with negative $\mu$ are known. However, for certain structures which are called left-handed materials (LHM), both the effective permittivity $\epsilon_{\text {eff }}$ and permeability $\mu_{\text {eff }}$ possess negative values. In such materials the index of refraction is less than zero, and therefore, phase and group velocity of an electromagnetic (EM) wave can propagate in opposite directions. This behavior leads to a number of interesting properties. ${ }^{3}$ The phenomena of negative index of refraction was first theoretically proposed by Veselago in $1968 .{ }^{4}$ Veselago also investigated various interesting optical properties of the negative index structures.

A negative permittivity medium can be obtained by arranging thin metallic wires periodically. ${ }^{5-10}$ This structure behaves like a high-pass filter which means that the effective permittivity will take negative values below the plasma frequency. On the other hand, a negative effective magnetic permeability medium is difficult to obtain. In 1999, Pendry et al. has suggested that an array of split ring resonators (SRRs) might exhibit a negative effective magnetic permeability for frequencies close to the resonance frequency of these structures. ${ }^{11}$ By combining these SRRs and thin wires, Smith and his co-workers reported the experimental demonstration of left-handed metamaterials. ${ }^{12}$ This was later followed by direct measurement of negative index of refraction, ${ }^{13}$ and analytical formulation of the left-handed medium. ${ }^{14}$ Also, the negative permittivity and permeability of CMM, as well as negative refraction index were calculated from the numerical data in Ref. 15. All of these measurements were performed in a waveguide chamber which

\footnotetext{
a) Author to whom correspondence should be addressed; electronic mail: bayindir@fen.bilkent.edu.tr

${ }^{b)}$ Present address: Institute of Physics, Slovak Academy of Sciences, Bratislava, Slovakia.

c) Also at: Research Center of Crete, IESL-FORTH, Heraklion, Crete, Greece.
}

limited one of the dimensions of the LHM structures to a maximum of three cells. ${ }^{16}$ Very recently, the fundamental properties of the LHMs were verified by the transfer matrix method (TMM), ${ }^{17} a b$ initio, ${ }^{18}$ the finite-element method, ${ }^{19}$ and finite-difference-time-domain ${ }^{20}$ simulations.

In this letter, we propose and demonstrate a type of CMM. The transmission spectra is obtained in free space which allows us to use CMM structures without any restrictions on the size of the structures. The CMM structures exhibit a passband within the stop bands of the SRRs and the thin wire structures. An improved version of the TMM is used to simulate our structures, and qualitative agreement with the experimental results is obtained.

We first constructed a CMM that consists of periodical arrangement of thin copper wires and SRRs. This configuration has a geometry which is similar to a previously reported structure. ${ }^{12,17}$ The details of the SRR structure is shown in Fig. 1(a). It consists of two rings separated by a gap, which is similar to the SRR structures in Refs. 16 and 17. As seen in Fig. 1(b), we first constructed the SRRs and the wires on

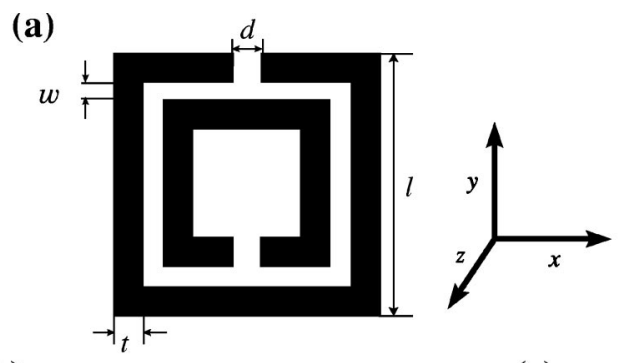

(b)

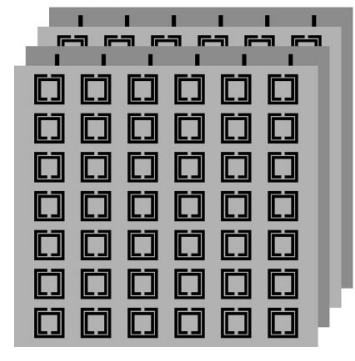

(c)

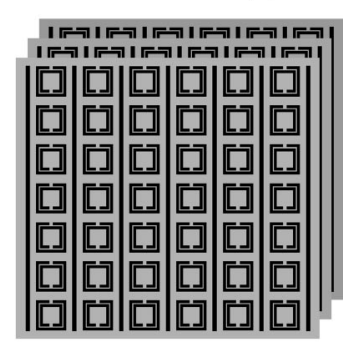

FIG. 1. (a) A single SRR with parameters $\ell=3 \mathrm{~mm}$ and $d=t=w$ $=0.33 \mathrm{~mm}$. (b) - (c) Schematic drawing of two different configurations of the composite medium consisting of thin wires and SRRs. 


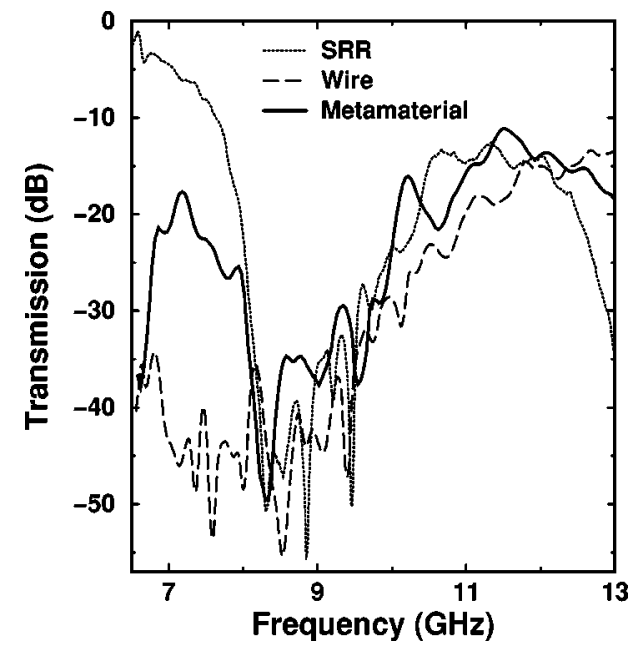

FIG. 2. Measured transmission spectra of thin wires, SRRs, and the composite structure with the first type of metamaterial configuration [Fig. 1(b)]. The transmission passband is observed due to negative values of the permittivity and the permeability.

separate boards and stacked them in a periodical arrangement.

We measured the transmission spectrum of a structure which is made by using $N_{x}=25, N_{y}=25$, and $N_{z}=20$ unit cells. Each unit cell consists of a copper wire and a SRR, and the dimensions of the unit cell are $a_{x}=5 \mathrm{~mm}, a_{y}$ $=3.63 \mathrm{~mm}$, and $a_{z}=5 \mathrm{~mm}$. The thickness and width of the thin copper wires are $30 \mu \mathrm{m}$ and $0.5 \mathrm{~mm}$. As shown in Fig. 1(a), we approximate the rings by squares of size $\ell$ $=3 \mathrm{~mm}$. The parameters of the SRR are $d=t=w$ $=0.33 \mathrm{~mm}$. The transmission measurements are performed in free space by using an HP 8510C network analyzer and microwave horn antennas. For all measurements, EM waves propagate along the $x$ direction. The electric field polarization is kept along the $y$ axis, and magnetic field polarization is kept along the $z$ axis. The thickness and the dielectric constant of the board are measured to be $0.45 \mathrm{~mm}$ and $\epsilon_{b}$ $=4.4$, respectively.

Figure 2 shows the measured transmission spectra of SRRs (dotted line), thin wires (dashed line), and the CMM (solid line). The SRR medium displays a stop band extending from 8.1 to $9.5 \mathrm{GHz}$ which is in agreement with the TMM simulations. ${ }^{17}$ The thin wire structure has a plasma frequency around $10 \mathrm{GHz}$. Although we were expecting the CMM transmission band to be at the same frequencies with the SRR stop band, we observed that the CMM transmission band shifted to lower frequencies $(6.7-8.1 \mathrm{GHz})$. Such a shift has also been reported in Ref. 16, and has been explained by the sensitivity of the mutual position of SRRs and wires with respect to each other.

To overcome this alignment problem, we constructed a second CMM structure [Fig. 1(c)]. In this configuration, we placed copper wires between the columns of the SRRs on the same board. This configuration has no alignment problems with the SRRs and thin wires, and can easily be fabricated at smaller scales. We then measured the response of EM to the CMM structure, which is made by $N_{x}=25, N_{y}=25$, and $N_{z}=20$ unit cells [Fig. 1(c)]. Each unit cell consists of a copper wire and a SRR, and the dimensions of the unit cell are $a_{x}=5 \mathrm{~mm}, a_{y}=3.63 \mathrm{~mm}$, and $a_{z}=6 \mathrm{~mm}$. As shown in

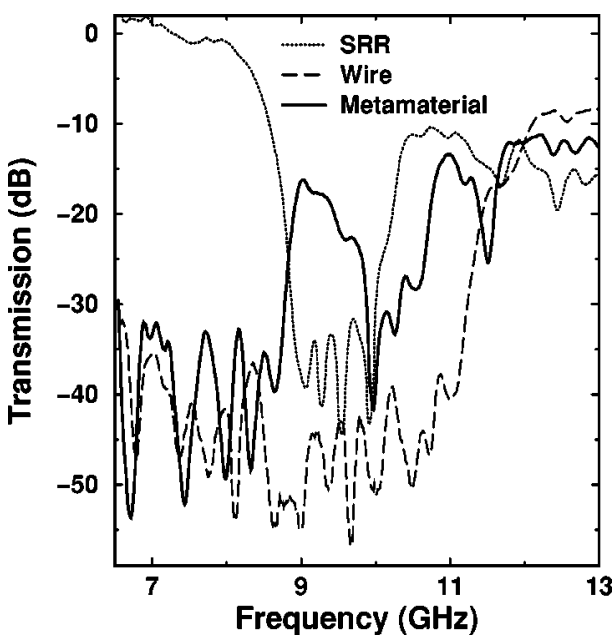

FIG. 3. Measured transmission spectra of thin wires, SRRs, and the composite structure with the second type of metamaterial configuration [Fig. 1(c)]. A transmission band is observed within the stop bands of wire and SRR structures.

Fig. 3, this CMM allows propagation of EM waves between 8.7 and $9.9 \mathrm{GHz}$. The CMM passband exactly coincides with the stop band of SRR. The wire structure also exhibits a stop band that covers the observed CMM passband. The peak transmission amplitude of the passband is $-16 \mathrm{~dB}$, which is higher than the $-24 \mathrm{~dB}$ peak amplitude reported in Ref. 16 .

We also performed numerical calculations for the CMMs. We used a modified version of the TMM code, ${ }^{21}$ which is recently developed to investigate the transmission and reflection properties of composite metamaterial structures. ${ }^{17}$ The main change from the standard algorithm commonly used to study photonic band gap materials ${ }^{22}$ is the faster normalization of the transmitted electromagnetic waves in the calculation of the transmission coefficient through the composite structures.

In order to calculate the transmission spectrum, the total volume of the system is divided into small cells and fields in each cell are coupled to those in the neighboring cells. We assume periodic boundary conditions in the directions parallel to the interfaces. Both SRRs and wires are located on the same dielectric board and the wire width is $0.66 \mathrm{~mm}$. The unit cell is $a_{x} \times a_{y} \times a_{z}=5 \times 3.66 \times 5 \mathrm{~mm}$. Each unit cell is discretized to $N_{x} \times N_{y} \times N_{z}=15 \times 11 \times 15$ mesh points. Ten unit cells are considered along the propagation direction, and periodic boundaries are supposed in $y$ and $z$ directions.

Figure 4 presents the calculated transmission spectra of the SRRs only (dotted line) and the CMMs structure (solid lines) corresponding to Fig. 1(c). The SRRs exhibits a forbidden band between 8.4 and $9.2 \mathrm{GHz}$, which is in good agreement with the measured results in Fig. 3. As the mesh length $(0.33 \mathrm{~mm}$ in the present simulations) defines the lower limit for the size of the components, we cannot simulate real thickness of the SRR and wire (which is only $0.03 \mathrm{~mm}$ ). We think that the resonance gap will shift slightly to higher frequencies in simulations made with more mesh points.

For the CMMs, we performed simulations for two different values of dielectric permittivity, namely $\epsilon_{\mathrm{CMM} 1}=1$ $+38000 i$, and $\epsilon_{\mathrm{CMM} 2}=-300000+588000 i$. It is observed that the larger imaginary part of the metallic permittivity gives higher transmission peak. When we take smaller 


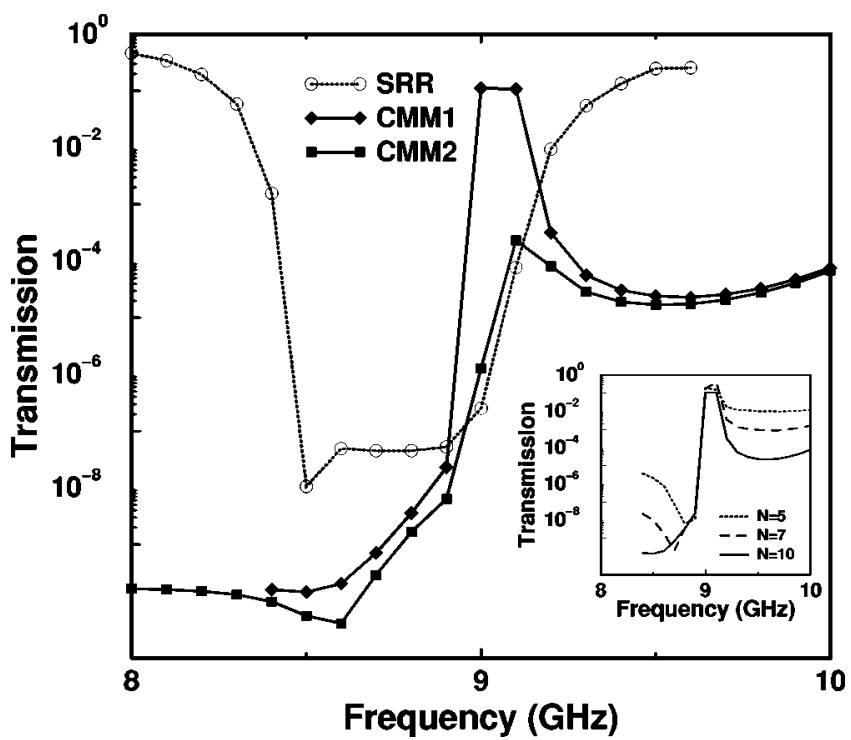

FIG. 4. Calculated transmission of electromagnetic waves through an array of SSRs (dotted line) and the composite metamaterials (solid lines) are shown in Fig. 1(c) for two different values of the metallic permittivity $\left(\epsilon_{\mathrm{CMM} 1}=1+38000 i\right.$, and $\left.\epsilon_{\mathrm{CMM} 2}=-300000+588000 i\right)$. Inset: Variation of the transmission spectra by increasing the number of unit cells along the propagation direction.

imaginary part (lossy materials), the transmission peak disappears. We also investigated how the number of unit cells along the propagation direction affects the peak transmission amplitude. As shown in the inset of Fig. 4, the peak disappears when the number of unit cells is decreased.

Our experimental and theoretical results on the CMMs clearly shows a transmission passband which is expected from the left-handed metamaterials. However, we still refrain from calling our CMM as a left-handed material. Further investigations, such as negative index measurements, has to be done with these structures for verification of the LHM behavior.

In conclusion, we report the free-space experimental measurement of composite metamaterials that consist of SRR and thin wire arrays. One of the structure exhibits a transmission passband, which indicates a possible lefthanded material property.
This work was supported by NATO Grant No. SfP971970, National Science Foundation Grant No. INT9820646, DARPA, NATO Grant Nos. PST. CLG. 978088, and NFS INT-0001236. P. M. thanks VEGA for partial financial support. Ames Laboratory is operated for the U.S. Department of Energy by Iowa State University under Contract No. W-7405-Eng-82.

${ }^{1}$ J. B. Pendry, Phys. World 14, 47 (2001).

${ }^{2}$ For a recent review, see articles in Photonic Crystal and Light Localization in the 21st Century, edited by C. M. Soukoulis (Kluwer, Dordrecht, 2001).

${ }^{3}$ J. B. Pendry, Phys. Rev. Lett. 85, 3966 (2000).

${ }^{4}$ V. G. Veselago, Sov. Phys. Usp. 10, 509 (1968).

${ }^{5}$ D. R. Smith, S. Schultz, N. Kroll, M. M. Sigalas, K. M. Ho, and C. M. Soukoulis, Appl. Phys. Lett. 65, 645 (1994).

${ }^{6}$ D. F. Sievenpiper, M. E. Sickmiller, and E. Yablonovitch, Phys. Rev. Lett. 76, 2480 (1996)

${ }^{7}$ J. B. Pendry, A. J. Holden, W. J. Stewart, and I. Youngs, Phys. Rev. Lett. 76, 4773 (1996).

${ }^{8}$ J. B. Pendry, A. J. Holden, D. J. Robbins, and W. J. Stewart, J. Phys.: Condens. Matter 10, 4785 (1998).

${ }^{9}$ D. R. Smith, D. C. Vier, W. J. Padilla, S. C. Nemat-Nasser, and S. Schultz, Appl. Phys. Lett. 75, 1425 (1999).

${ }^{10}$ B. Temelkuran, M. Bayindir, E. Ozbay, J. P. Kavanaugh, M. M. Sigalas, and G. Tuttle, Appl. Phys. Lett. 78, 264 (2001).

${ }^{11}$ J. B. Pendry, A. J. Holden, D. J. Robbins, and W. J. Stewart, IEEE Trans. Microwave Theory Tech. 47, 2075 (1999).

${ }^{12}$ D. R. Smith, W. J. Padilla, D. C. Vier, S. C. Nemat-Nasser, and S. Schultz, Phys. Rev. Lett. 84, 4184 (2000).

${ }^{13}$ R. A. Shelby, D. R. Smith, and S. Schultz, Science 292, 77 (2001).

${ }^{14}$ D. R. Smith and N. Kroll, Phys. Rev. Lett. 85, 2933 (2000).

${ }^{15}$ D. R. Smith, S. Schultz, P. Markos, and C. M. Soukoulis, Phys. Rev. B 65, 195104 (2002).

${ }^{16}$ R. A. Shelby, D. R. Smith, and S. C. Nemat-Nasser, Appl. Phys. Lett. 78, 489 (2001).

${ }^{17}$ P. Markos and C. M. Soukoulis, Phys. Rev. B 65, 033401 (2002); Phys. Rev. E 65, 036622 (2002).

${ }^{18}$ T. Weiland, R. Schuhmann, R. B. Greegor, C. G. Parazzoli, A. M. Vetter, D. R. Smith, D. C. Vier, and S. Schultz, J. Appl. Phys. 90, 5419 (2001).

${ }^{19}$ C. Caloz, C. C. Chang, and T. Itoh, J. Appl. Phys. 90, 5483 (2001).

${ }^{20}$ R. W. Ziolkowski and E. Heyman, Phys. Rev. E 64, 056625 (2001).

${ }^{21}$ J. B. Pendry, A. MacKinnon, and P. J. Roberts, Proc. R. Soc. London, Ser. A 437, 67 (1992).

${ }^{22}$ J. B. Pendry, J. Mod. Opt. 41, 209 (1994). 